\title{
A CLASS OF ERROR TOLERANT PATTERN DISCRIMINATION FUNCTIONS*
}

\author{
William A. Porter \\ Computer, Information and Control Engineering Program, The University of Michigan. \\ Ann Arbor, MI 28104, U.S.A.
}

(Received 12 January 1976 and in revised form 1 July 1976)

\begin{abstract}
A general pattern recognition problem is posed in Hilbert space. Two new solutions are then given and it is shown that the sensitivity of the pattern recognition functions to pattern perturbation can be a priori controlled. A series of examples demonstrate the principal results in a variety of settings.
\end{abstract}

Pattern recognition Multilinearity Hilbert space Functional sensitivity

\section{INTRODUCTION}

The term pattern recognition is used in a diversity of settings $^{(1,4)}$ and hence it is only proper that we delineate the problem to be considered here. For this we choose a hypothetical automated manufacturing application as a vehicle for the discussion. In short, consider a machining unit which is presented with a parts flow drawn from a large but finite number of distinct parts. Each part must be recognized, oriented, secured and machined in an appropriate way.

For simplicity, suppose that each part can be physically oriented and secured in the field of view of a set of optical scanners. The scanner outputs constitute a time-varying set of patterns from which the part must be identified, and oriented for machining. Our interest here is in the part identification problem which has an obvious pattern recognition flavor.

To model our problem in mathematical form consider first a discrete set of optical sensors with $n$ outputs $\left\{x_{i}\right\}$. At the instant, $t$, we may view $\left\{x_{i}(t)\right\}$ as an $n$-tuple or as a geometric array. For convenience we adopt the $n$-tuple viewpoint and let $x(t)=$ $\left(x_{1}(t), x_{2}(t), \ldots, x_{n}(t)\right)$ be the instantaneous pattern. The case where the optical scan is continuous in a spatial variable is also of interest and we shall use the notation $x(\alpha, t)$ to denote the instantaneous scan function as it depends on the spatial variable $\alpha$. Even more generally $\alpha$ could be interpreted as a triplet of 3 spatial variables in which case the scanner would have three dimensional perception.

In any of the above cases a static pattern recognition problem can be easily formulated. For each of the possible parts we assume an a priori pattern which may be viewed as a signature of the part. We have then a set of signatures, $\Gamma=\left\{\gamma_{1}, \ldots, \gamma_{m}\right\}$, where each $\gamma_{j} \in \Gamma$ is the same type of entity as the scanner

* Sponsored in part by the Air Force Office of Scientific Research under Grant AFOSR 73-2427. output. That is if $x(t)$ is an $n$-tuple then each $\gamma_{j}$ is also an $n$-tuple.

More precisely, let $X$ be the universe of admissible patterns. Let $Y$ be a set containing at least $m$ distinct points $\left\{y_{1}, \ldots, y_{m}\right\}$.

Problem 1. Find a function (or functions) $f: X \rightarrow Y$ such that

$$
f\left(\gamma_{i}\right)=y_{i} \quad i=1,2, \ldots, m .
$$

Problem 1, which has been studied in a variety of settings, provides a function with a distinct output for each of the distinct $\gamma_{i} \in \Gamma$. It does not, however, take into account the effects of noise or variations in the patterns due, for example, to manufacturing tolerance. One way of modeling this would be to equip $X$ and $Y$ with a topology and then try to solve Problem 2, namely:

Problem 2. Find a function $f: X \rightarrow Y$ such that $f\left(\gamma_{i}\right)=y_{i}, i=1,2, \ldots, m$ and if $w$ is close to $\gamma_{i}$, then $f(w)$ is close to $f\left(\gamma_{i}\right)$.

It is noted that existing minimum distance, maximum probability, maximum dot product and committee machine techniques ${ }^{(4)}$ generate solutions to Problem 2. In the present study we develop a linear and a polynomic solution neither of which appears to have been considered by other authors. Both solutions present a function which has the $f\left(\gamma_{i}\right)=y_{i}$, $i=1, \ldots, m$ property plus its derivative (Frechet) $f^{\prime}$ satisfies $f^{\prime}\left(\gamma_{i}\right)=0$. The net effect is that $f^{\prime}$ has zero first order sensitivity to additive disturbances. Of course this results in very effective suppression of small errors.

In some applications it might actually be desirable to have a large derivative at $\gamma_{i}$. Indeed, in the manufacturing setting, the errors may be due to inaccurate part alignment and a high sensitivity to this could be useful in the alignment procedure. The techniques described in the following can set the derivative value arbitrarily. We consider only $f^{\prime}\left(\gamma_{i}\right)=0$ for simplicity of exposition. 
The present paper develops the analytic properties of the recognition functions in question. In a subsequent paper $^{(8)}$ the results of an extensive computer simulation of these functions and such conventional recognition functions as maximum probability are reported. In these computer studies the Highleyman alphabetic data bank ${ }^{(5)}$ and standard multispectral environmental data ${ }^{(7)}$ were utilized.

\section{SOME PRELIMINARIES}

The discussion of the first Section must be given a precise mathematical interpretation before the stated problems can be solved. If the analysis is to be applicable to a variety of settings such as discrete pattern tones, continuous pattern tones, multiple continuous and discrete spatial variables, and probabilistic effects, then the mathematical format must be abstract enough to encompass these variations.

The mathematical structure adopted here is the abstract Hilbert space. In solving the stated problems we shall move forward on an abstract level, intermixing examples to illustrate and motivate the development. In this spirit we first consider three examples.

Let $H$ denote a Hilbert space with inner product $\langle\cdot, \cdot\rangle^{(3)}$. The scalar field is denoted by $F$ while $\Gamma \subset H$ is the pattern set.

Example 1. For continuous spatial scanning (in one variable) suppose $[a, b]$ is the spatial domain. A function $x$ is an element of $H$ if $x$ is real valued and

$$
\|x\|^{2}=\int_{a}^{b}|x(\alpha)|^{2} \mathrm{~d} \alpha<\infty .
$$

It is well known that $H$ is a Hilbert space. We also note that $H$ has a dot product. That is, a bilinear map to the scalars

$$
\langle x, y\rangle=\int_{a}^{b} x(\alpha) y(\alpha) \mathrm{d} \alpha,
$$

satisfying the usual inner product axioms ${ }^{(3)}$.

Example 2. For discrete spatial scanning suppose we have $n$ scan points in which case $H$ is the set of all real $n$-tuples, i.e. $R^{n}$. We note that $H=R^{n}$ is a Hilbert space over the real field and has a dot product computed by

$$
\langle x, y\rangle=\sum_{i=1}^{n} x_{i} y_{i}
$$

where $x=\left(x_{1}, \ldots, x_{n}\right)$ and $y=\left(y_{1}, \ldots, y_{n}\right)$ are elements of $H$.

In both of these examples the scalar field consisted of the real numbers. However if the pattern was quantitized into white, black, and a finite number of grey tones, it is more natural to use a finite field. Both of the previous examples can easily be modified. For emphasis, we earmark the quantitized scan case by a separate example.

Example 3. Let $\Omega$ consist of all $n$-tuples with entries from the set $\{0,1,2\}=F$. Using $\bmod 3$ addition and scalar multiplication the well known fact that $F$ is a linear space over $\Omega$ follows easily. It is also clear that the bilinear map

$$
\langle x, y\rangle=\sum_{i=1}^{n} x_{i} y_{i},
$$

can be computed. This map, however, is not a legitimate dot product on $F$ if the additions and multiplications are mod 3 . Noting that $F^{n} \subset R^{n}$ we use the above bilinear map computing it in $R^{n}$ instead of $F^{n}$. Of course $\langle$,$\rangle maps into the reals instead of F$ and as such is not truely a dot product on $F^{n}$ although we shall refer to it as such.

\section{SOLVING PROBLEM}

Returning now to Problem 1 we may formalize as follows:

Problem 1. Let $H$ denote a Hilbert space with an inner product function $\langle\cdot, \cdot\rangle$. Let $\Gamma=\left\{\gamma_{1}, \ldots, \gamma_{m}\right\} \subset H$ be a finite set of distinct elements. Let $\left\{y_{1}, \ldots, y_{m}\right\}$ be any set of distinct symbols. Construct a function $f$ such that

$$
y_{i}=f\left(\gamma_{i}\right) \quad i=1,2, \ldots, m .
$$

The posed problem has numerous solutions and we shall mention only two here. The first solution is multilinear. Using the notation $\|z\|^{2}=\langle z, z\rangle$ we form the functionals

$$
\psi_{i}(x)=\prod_{k \neq i}^{m} \frac{\left\langle x-\gamma_{k}, \gamma_{i}-\gamma_{k}\right\rangle}{\left\|\gamma_{i}-\gamma_{k}\right\|^{2}} i=1, \ldots, m .
$$

It is easily seen that

$$
\psi_{i}\left(\gamma_{j}\right)=\left\{\begin{array}{ll}
0 & j \neq i \\
1 & j=i
\end{array} \quad i=1, \ldots, m\right.
$$

and hence

Lemma 1. The function

$$
f(x)=\sum_{i=1}^{m} y_{i} \psi_{i}(x)
$$

is a solution to Problem 1.

Example 4. In the context of Example 1 let $\Gamma=$ $\left\{\gamma_{1}, \gamma_{2}\right\}$ where for instance, $\gamma_{1}(\alpha)=k$ and $\gamma_{2}(\alpha)=l \alpha$. Then

$$
\begin{aligned}
& \psi_{1}(x)=\frac{\int_{a}^{b}[x(\alpha)-l \alpha][k-l \alpha] \mathrm{d} \alpha}{\int_{a}^{b}[k-l \alpha]^{2} \mathrm{~d} \alpha} \\
& \psi_{2}(x)=\frac{\int_{a}^{b}[x(\alpha)-k][l \alpha-k] \mathrm{d} \alpha}{\int_{a}^{b}[k-l \alpha]^{2} \mathrm{~d} \alpha} .
\end{aligned}
$$

The discrete scanning of Example 2 exhanges summations for the above integrals. As for the finite tone setting, we treat that separately since there is some concern over the validity of the inner product. 
Example 5. Here we take $\Gamma=\left\{\gamma_{1}, \gamma_{2}, \gamma_{3}\right\}$ where, for illustrative purposes $F=\{0,1,2\}$ and

$$
\begin{aligned}
& \gamma_{1}=(0,1,1,2,1) \\
& \gamma_{2}=(1,1,1,0,1) \\
& \gamma_{3}=(1,0,1,1,0),
\end{aligned}
$$

using nonfinite arithmetic we compute

$$
\begin{aligned}
& \Delta_{12}=\gamma_{1}-\gamma_{2}=(-1,0,0,2,0) \\
& \Delta_{13}=\gamma_{1}-\gamma_{3}=(-1,1,0,1,1) \\
& \Delta_{23}=\gamma_{2}-\gamma_{3}=(0,1,0,-1,1)
\end{aligned}
$$

of course $\Delta_{i j}=-\Delta_{j i}$ and from the above

$$
\begin{aligned}
& \left\|\Delta_{12}\right\|^{2}=5 \\
& \left\|\Delta_{13}\right\|^{2}=4
\end{aligned}
$$

and

$$
\left\|\Delta_{23}\right\|^{2}=3 \text {. }
$$

Let $x=\left(x_{1}, \ldots, x_{5}\right) \in R^{5}$ be arbitrary. Then, still using nonfinite arithmetic,

$$
\begin{aligned}
\left\langle x-\gamma_{2}, \gamma_{1}-\gamma_{2}\right\rangle & =-\left(x_{1}-1\right)+2\left(x_{4}\right) \\
\left\langle x-\gamma_{3}, \gamma_{1}-\gamma_{3}\right\rangle=- & -\left(x_{1}-1\right) \\
& +\left(x_{2}\right)+\left(x_{4}-1\right)+\left(x_{5}\right)
\end{aligned}
$$

Since $\left\|\Delta_{12}\right\| \cdot\left\|\Delta_{13}\right\|=20$ we have

$$
\psi_{1}(x)=\frac{\left(2 x_{4}-x_{1}+1\right)\left(x_{4}+x_{5}+x_{2}-x_{1}\right)}{20} .
$$

Similar computations identify $\psi_{2}, \psi_{3}$ as

$$
\begin{array}{r}
\psi_{2}(x)=\left(x_{1}-2 x_{4}+4\right)\left(x_{2}-x_{4}+x_{5}+1\right) / 15 \\
\begin{array}{r}
\psi_{3}(x)=\left(x_{1}-x_{2}-x_{4}-x_{5}+4\right) \\
\times\left(-x_{2}+x_{4}-x_{5}+2\right) / 12 .
\end{array}
\end{array}
$$

It is easily verified that $\psi_{i}\left(\gamma_{j}\right)=\delta_{i j}$ and hence the solution to Problem 1, identified in (2), can be constructed. Moreover if the set $\left\{y_{i}\right\}$ consists of distinct code words the function $f$ outputs in transmittable form.

Suppose $G=\{g: g(\Gamma)=0\}$, then if $g \in G$, and $Y$ is a linear space, clearly $f+g$ is another solution to Problem 1. The converse is also true and we have:

Lemma 2. Let $Y$ be a linear space. Then $\hat{f}$ is a solution to Problem 1 if and only if $\hat{f}=f+g$ for some $g \in G$.

Before proceeding to Problem 2, we shall identify one other specific solution to Problem 1. For this we assume that the set $\Gamma$ is linearly independent. Then there exists a unique set $\Gamma^{+}=\left\{\gamma_{1}^{+}, \ldots \gamma_{m}^{+}\right\}$with the properties

$$
\begin{gathered}
\operatorname{Span}\left(\Gamma^{+}\right)=\operatorname{Span}(\Gamma) \\
\left\langle\gamma_{j}^{+}, \gamma_{i}\right\rangle=\delta_{i j} .
\end{gathered}
$$

The set $\Gamma^{+}$is called the dual of $\Gamma$. It may be computed by first inverting the grammian matrix

$$
M=\left[\left\langle\gamma_{i}, \gamma_{j}\right\rangle\right],
$$

which is nonsingular by virtue of the linear independence of $\Gamma^{(3)}$ and then using the row entries of $M^{-1}$ as coefficients in the following way. If $\left(\alpha_{i 1}\right.$, $\left.\alpha_{i 2}, \ldots, \alpha_{i m}\right)$ is the $i^{\text {th }}$ row of $M^{-1}$ then

$$
\gamma_{i}^{+}=\sum_{j=1}^{m} x_{i j} \gamma_{j}, i=1, \ldots, m
$$

Lemma 3. Let $Y$ be a linear space and $\Gamma$ be linearly independent in $\mathrm{H}$. Then

$$
g(x)=\sum_{i=1}^{m} y_{i}\left\langle\gamma_{i}^{+}, x\right\rangle
$$

is a linear solution to Problem 1.

That Lemma 3 is correct is easily verified using equation (3). In the context of Examples 3 and 5 the dot products and matrix inversions are all done in $R^{3}$. For instance in the context of Example 4 the Grammian is $2 \times 2$ and has the numerical form (take $a=0, b=1$ for convenience)

$$
M=\left[\begin{array}{ll}
k^{2} & \frac{k l}{2} \\
\frac{k 1}{2} & \frac{l^{2}}{3}
\end{array}\right] .
$$

which has the inverse

$$
M^{-1}=(k 1)^{-2}\left[\begin{array}{cc}
41^{2} & -6 k 1 \\
-6 k 1 & 12 k^{2}
\end{array}\right] .
$$

Using the rows of $M^{-1}$ as indicated above, we find

$$
\begin{aligned}
& \gamma_{1}^{+}(\alpha)=2 k^{-1}\{2-3 \alpha\} \\
& \gamma_{2}^{+}(\alpha)=6 l^{-1}\{-1+2 \alpha\}
\end{aligned} \quad \alpha \in[0,1] .
$$

To complete the construction suppose that $y_{1}=(1,0)$ and $y_{2}=(0,1)$. Then using the inner product we see that $g$ outputs a tuplet computed by the equality

$$
\begin{aligned}
g(x)=\left(2 k^{-1} \int_{0}^{1}\right. & \{2-3 \alpha) x(\alpha) \mathrm{d} x, \\
& \left.\times 6 l^{-1} \int_{0}^{1}\{-1+2 \alpha\} x(\alpha) \mathrm{d} \alpha\right) .
\end{aligned}
$$

In our examples we have stressed what could be construed as a time domain construction of the pattern identification function. However, since the Fourier transform is a unitary operator on $L_{2}(0, \infty)$, the frequency domain is never far from hand. Indeed, using Parseval's theorem, the functions of Lemmas 1 and 2 can both be interpreted in the frequency domain setting. Their implementation in that setting could involve, for example, an optical filter. In this case Lemmas 1 and 2 specify the spatial filter in the optical processor $^{(2)}$

\section{SOLVING PROBLEM 2}

We now take up the question of solutions to Problem 2. For this we shall make use of the concept 
of abstract differentiation (see [9]). Let $\mathbf{H}$ denote a Hilbert space and $\psi$ a (Frechet) scalar-valued function. A scalar-valued function $\Psi$ is said to be the derivative (Frechet) of $\psi$ at $u_{0}$ if

$$
\lim _{\|v\| \rightarrow 0} \frac{\left\|\psi\left(u_{0}+v\right)-\psi\left(u_{0}\right)-\Psi\left[u_{0}, v\right]\right\|}{\|v\|},
$$

where $\Psi\left[u_{0}, \cdot\right]$ is linear.

Now suppose $\psi_{i}$ is any function satisfying $\psi_{i}\left(\gamma_{j}\right)=$ $\delta_{i j}, i j=1, \ldots, m$ and having the derivative $\Psi_{i}$ at $\gamma_{i}$. Then we form

$$
h_{i}(u)=\left\{1-2 \Psi_{i}\left[\gamma_{i}, u-\gamma_{i}\right]\right\} \psi_{i}^{2}(u),
$$

and denote its Frechet derivative by $H_{i}$.

Lemma 4.

$$
\begin{array}{cc}
\text { (i) } h_{i}\left(\gamma_{j}\right)=\delta_{i j} & i, j=1,2, \ldots, m \\
\text { (ii) } H_{i}\left[x_{j}, \cdot\right]=0 & i, j=1,2, \ldots, m .
\end{array}
$$

Proof. Part (i) follows from inspection. For part (ii) we differentiate equation (5), using the chain rule for Frechet derivatives. ${ }^{(9)}$

$$
\begin{aligned}
H_{i}[u, \delta v]= & -2 \Psi_{i}[u, \delta v] \psi_{i}^{2}(u) \\
& +\left\{1-2 \Psi_{i}\left[u, u-\gamma_{i}\right]\right\} 2 \psi_{i}(u) \Psi_{i}[u, \delta v],
\end{aligned}
$$

when $u=\gamma_{j}$ we have $H_{i}\left[\gamma_{j}, \delta v\right]=0$. While for $u=\gamma_{i}$

$$
H_{i}\left[\gamma_{i}, \delta v\right]=-2 \Psi_{i}\left[\gamma_{i}, \delta v\right]+\{1\} 2 \Psi_{i}\left[\gamma_{i}, \delta v\right]=0
$$

which completes the proof.

Theorem 1. Let $\left\{\psi_{i}\right\}$ be any set of differentiable functions satisfying $\psi_{i}\left(\gamma_{j}\right)=\delta_{i j}$. Let $\left\{h_{i}\right\}$ be computed as in equation (5). Then

$$
\widehat{f}(u)=\sum_{i=1}^{m} y_{i} h_{i}(u)
$$

is a solution to Problem 2.

The solution $\hat{f}$ of equation (6) is unique to within the addition of an arbitrary function which is zero on $\Gamma$ and has a zero derivation on this set. We also note that Theorem 1 can be implemented using the functionals computed in equation (1) or (4). In both cases a more explicit form of the $\left\{h_{i}\right\}$ is forthcoming.

Consider first the case where $\psi_{i}$ is computed as in equation (1). Differentiating by parts it follows readily that

$$
\begin{aligned}
\Psi_{i}[u, \delta v]= & \left\{\sum_{j \neq i}\left\langle\delta v, \gamma_{i}-\gamma_{j}\right\rangle\right. \\
& \left.\prod_{k \neq i, j}\left\langle u-\gamma_{k}, \gamma_{i}-\gamma_{k}\right\rangle\right\} \\
& \div \prod_{j \neq i}\left\langle\gamma_{i}-\gamma_{j}, \gamma_{i}-\gamma_{j}\right\rangle,
\end{aligned}
$$

hence

$$
\Psi_{i}\left[\gamma_{i}, u-\gamma_{i}\right]=\sum_{j \neq i}\left(\frac{\left\langle u-\gamma_{i}, \gamma_{i}-\gamma_{j}\right\rangle}{\left\langle\gamma_{i}-\gamma_{j}, \gamma_{i}-\gamma_{j}\right\rangle}\right) .
$$

It follows then that

$$
h_{i}(u)=\left\{1-2 \sum_{j \neq i} \frac{\left\langle u-\gamma_{i}, \gamma_{i}-\gamma_{j}\right\rangle}{\left\langle\gamma_{i}-\gamma_{j}, \gamma_{i}-\gamma_{j}\right\rangle}\right\} \psi_{i}^{2}(u) .
$$

It is not difficult to verify directly that the $\left\{h_{i}\right\}$ of equation (7) satisfy the conditions of Lemma 4.

Suppose now that $\Gamma$ is linearly independent, then the functionals $\psi_{i}(u)=\left\langle\gamma_{i}^{+}, u\right\rangle$ of equation (4) satisfy the conditions of Theorem 1. Moreover

$$
\Psi_{i}[u, \delta v]=\left\langle\gamma_{i}^{+}, \delta v\right\rangle i=1, \ldots, m
$$

hence in equation (5) we have

$$
h_{i}(u)=\left\{1-2\left\langle\gamma_{i}^{+}, u-\gamma\right\rangle\right\}\left\langle\gamma^{+}, u\right\rangle^{2} i=1, \ldots, m .
$$

As before, the properties of Lemma 4 can be verified directly using the functional defined in equation (8).

Corollary. If $\Gamma$ has no duplicate points, then the functionals of equation (7) together with equation (6) solve Problem 2. If $\Gamma$ is linearly independent then the functionals of equation (8) used in equation (6) solve Problem 2.

Example 6. Let us return to Example 5 and consider the function $\psi_{1}$ of that example. By a direct computation we see that

$$
\begin{aligned}
& \psi_{1}(x+v) \\
& \left(2 x_{4}+2 v_{4}-x_{1}-v_{1}+1\right) \\
& =-\frac{\left(x_{4}+v_{4}+x_{5}+v_{5}+x_{2}+v_{2}-x_{1}-v_{1}\right)}{20} \\
& =\psi_{1}(x)+\frac{\left(2 x_{4}-x_{1}+1\right)\left(v_{4}+v_{5}+v_{2}-v_{1}\right)}{20} \\
& +\frac{\left(2 v_{4}-v_{1}\right)\left(x_{4}+x_{5}+x_{2}-x_{1}\right)}{20} \\
& +\frac{\left(2 v_{4}-v_{1}\right)\left(v_{4}+v_{5}+v_{2}-v_{1}\right)}{20},
\end{aligned}
$$

and hence

$$
\begin{aligned}
20 \Psi_{1}[x, v]= & \left(2 x_{4}-x_{1}+1\right) v_{5} \\
& +\left(2 x_{5}+4 x_{4}+2 x_{2}-3 x_{1}+1\right) v_{4} \\
& +\left(2 x_{4}-x_{1}+1\right) v_{2} \\
& +\left(-x_{5}-3 x_{4}-x_{2}+2 x_{1}-1\right) v_{1}
\end{aligned}
$$

Using $\gamma_{1}=(0,1,1,2,1)$ we have then

$$
\begin{aligned}
20 \Psi_{1}\left[\gamma_{1}, u-\gamma_{1}\right]= & 5\left(u_{5}-1\right)+13\left(u_{4}-2\right) \\
& +5\left(u_{2}-1\right)-9\left(u_{1}-0\right) \\
= & 5 u_{5}+13 u_{4}+5 u_{2}-9 u_{1}-36,
\end{aligned}
$$

where $u=\left(u_{1}, \ldots, u_{5}\right)$. Now using (5) we have

$$
\begin{aligned}
h_{1}(u)= & \left\{\frac{46+9 u_{1}-5 u_{2}-13 u_{4}-5 u_{5}}{10}\right\} \times \\
& \left\{\frac{\left(2 u_{4}-u_{1}+1\right)\left(u_{5}+u_{4}+u_{2}-u_{1}\right)}{20}\right\}^{2} .
\end{aligned}
$$


By direct computation it can be verified that

$$
\begin{gathered}
h_{1}\left(\gamma_{j}\right)=\delta_{1 j} \\
\left(\frac{\partial h_{1}}{\partial u_{1}}\right)\left(\gamma_{j}\right)=0 \quad i=1, \ldots, 5 ; j=1,2,3,
\end{gathered}
$$

hence $h_{1}$ has the desired properties.

\section{DISCUSSION}

There are several points of interest in our solution to Problem 2. First, let us focus on equation (8) and note that it can be rewritten

$$
h_{i}(u)=1-3\left[\left\langle\gamma_{i}^{+}, u\right\rangle-1\right]^{2}-2\left[\left\langle\gamma_{i}^{+}, u\right\rangle-1\right]^{3} \text {. (9) }
$$

That is, if $p(x)=1-3(x-1)^{2}-2(x-1)^{3}$ then $h_{i}(u)=$ $p\left(\left\langle\gamma_{i}^{+}, u\right\rangle\right)$. Moreover, $p(x)$ is the unique polynomial of order $\leq 3$ satisfying

$$
\begin{array}{ll}
p(0)=0 & p^{\prime}(0)=0 \\
p(1)=1 & p^{\prime}(1)=0 .
\end{array}
$$

From this it is clear how one can suppress any finite number of Frechet derivatives. For example if $p$ : $R \rightarrow R$ satisfies

$$
p(0)=p^{\prime}(0)=p^{\prime \prime}(0)=p^{\prime}(1)=p^{\prime \prime}(1)=0 p(1)=1 .
$$

Then $h_{i}(u)=p\left(\left\langle\gamma_{i}^{+}, u\right\rangle\right)$ provides (using equation 6) a function such that $f\left(\gamma_{i}\right)=y_{i}$ and $f^{\prime}\left(\gamma_{i}\right)=f^{\prime \prime}\left(\gamma_{i}\right)=0$, $i=1, \ldots, m$.

We take note also of the fact that the solution utilizing equation (8) is quasi-linear. Indeed, if

$$
x=\sum_{i=1}^{m} \alpha_{i} \gamma_{i}
$$

then

$$
f(x)=\sum_{i=1}^{m} p\left(\alpha_{i}\right) y_{i}
$$

where $p$ is the third-order polynomial indicated above.

The concept of a multilinear operator is also relevant. Suppose $W$ maps $X^{n}$ into $Y$ where both are linear spaces. We say that $W\left[x_{1}, x_{2}, \ldots, x_{n}\right]$, is $n$-linear if $W$ is linear, in every argument. For example

$$
\begin{aligned}
W\left[\alpha x_{1}^{\prime}+\beta x_{1}^{\prime \prime}, x_{2}, \ldots, x_{n}\right] & =\alpha W\left[x_{1}^{\prime}, x_{2}, \ldots, x_{n}\right] \\
& +\beta W\left[x_{1}^{\prime \prime}, x_{2}, \ldots, x_{n}\right],
\end{aligned}
$$

holds for all $x_{1}^{\prime}, x_{1}^{\prime \prime}, x_{2}, \ldots, x_{n}, \alpha, \beta$. If $k(x)=W[x$, $x, \ldots, x]$ where $W$ is $n$-linear, then $k$ is said to be $n$-power, obviously $k(\lambda x)=\lambda^{n} k(x)$. Finally, a finite sum of $n$-power operators is said to be polynomic with the order of the operator being the largest multiplicity of the $n$-power operators in its sum. Notice that $n=0$ is a constant vector, and $n=1$ is a linear operator.

From equation (8) it follows easily that the second solution to Problem 2 is a polynomic operator of order 3. The first solution of Problem 2 is a polynomic operator of order $2 m-1$. Examples can be constructed which have polynomic solutions of order $n$ for any $3<n<2 m-1$.

\section{SUMMARY}

One thrust of the development is to demonstrate that a broad spectrum of pattern recognition problems can be modeled in Hilbert space. In this setting pattern recognition becomes a function design problem. It was shown that polynomic functions provide a convenient construction technique wherein the derivatives of the recognition functions can be a priori controlled.

While a hypothetical automated manufacturing context was used to motivate the development an equally convenient motivation comes from the area of alphabetic character recognition ${ }^{(5.6)}$. In this setting $\Gamma$ represents the alphabetic symbols. Problem 2 is relevant to identifying these symbols with distortion caused by scale factor or difference in type face and hand written variation.

Another motivating example arises in the processing of multispectral data relevant to remote agricultural crop survey ${ }^{(7)}$. In this setting $\Gamma$ consists of the multispectral signatures of various crops as corrupted by soil, moisture, seasonal, cloud cover and other variables.

Our attention here has focused on the abstract definitions and concepts involved. This has been accompanied by an extensive simulation using both the Highleyman data bank ${ }^{(5)}$ of hand written characters and a standard multispectral data bank taken over California's Imperial Valley. In these simulations the present pattern recognition functions were compared with minimum distance and maximum probability pattern recognizers. The simulation results and associated analysis are in preparation as a separate study ${ }^{(8)}$

\section{REFERENCES}

1. L. N. Kanal (Ed.), Pattern Recognition, Thompson, Washington, D.C. (1968).

2. A. Vanderlugt, Character Reading by Optical Spatial Filtering in Optical Electro-Optical Information Processing (Ed. Tippet) MIT Press, (1965).

3. W. A. Porter, Modern Foundations of Systems Engineering, Macmillan, NY (1966).

4. H. C. Andrews, Mathematical Techniques in Pattern Recognition, Wiley-Interscience, NY (1972).

5. W. H. Highleyman, An Analog method for character recognition, Trans. IRE, 10, (50). No. 1 (1961).

6. J. R. Ullmann and P. A. Kidd, Recognition experiments with typed numerals from envelopes in the mail, Pattern Recognition, 1, 273-289 (1969).

7. R. B. Crain, A study of techniques for processing multispectral scanner data. Report 31650-155-T. Environmental Research Institute of Michigan (1973).

8. W. A. Porter. A comparison of selected pattern recognition functions, Pattern Recognition 9. 59-64 (1977).

9. L. Liusternik and V. Sobolev, Elements of finctional analysis, Fredrick Unger, NY (1961). 
About the Author-William A. Porter was born in South Haven, MI, on 11 December, 1934. He attended the Michigan Technological University and subsequently the University of Michigan, Ann Arbor, where he received the doctorate degree in electrical engineering in 1961.

$\mathrm{He}$ is presently Professor of Electrical and Computer Engineering at the University of Michigan, Ann Arbor. His research and teaching interests currently include pattern recognition, neuromuscular systems, non-self-adjoint resolvant operator theory and assorted applications. He has authored two texts and numerous research publications. He is currently serving as a Consultant with Minneapolis Honeywell in the area of sensitivity reducing design procedures. 